\title{
BMJ Open Assessment for the possibility of a first night effect for wrist actigraphy in adolescents
}

\author{
Teresa Arora, ${ }^{1}$ Omar M Omar, ${ }^{1}$ Shahrad Taheri ${ }^{1,2}$
}

To cite: Arora T, Omar OM, Taheri S. Assessment for the possibility of a first night effect for wrist actigraphy in adolescents. BMJ Open 2016;6: 012172.

doi:10.1136/bmjopen-2016012172

- Prepublication history for this paper is available online. To view these files please visit the journal online (http://dx.doi.org/10.1136/ bmjopen-2016-012172).

Received 6 April 2016 Revised 12 June 2016 Accepted 5 August 2016
CrossMark

\footnotetext{
${ }^{1}$ Department of Medicine and Clinical Research Core, Weill Cornell Medicine in New York USA, Doha, Qatar

2Department of Medicine,

King's College London,

London, UK
}

Correspondence to Professor Shahrad Taheri; staheri@me.com

\section{ABSTRACT}

Objectives: Evidence of a 'first night effect' has been documented for polysomnography. The possibility of this has not been previously assessed in wrist actigraphy, yet may have important implications for the study design of future sleep research. We sought to examine potential evidence of a 'first night effect' for wrist actigraphy in adolescents across weekdays and weekend nights for multiple sleep outcomes.

Design: 3-year prospective cohort study (Midlands Adolescent Schools Sleep Education Study).

Setting: 8 secondary schools in the Midlands region of the UK.

Participants: Adolescents (aged 11-13 years at baseline) were recruited to the study and were requested to wear a wrist actigraph for 7 consecutive days/nights at baseline and then annually for 2 years during the second term of the academic year.

Primary outcome measures: We compared multiple sleep outcomes (total sleep time, wake after sleep onset, sleep efficiency, sleep onset latency, number of awakenings, length of awakenings, sleep onset time) when the device was worn on a weekday and weekend and compared these to other nights to identify possible evidence of a 'first night effect' for wrist actigraphy.

Results: No significant differences were found between any sleep outcomes when the first night of wrist actigraphy was on a weekday compared with other weekdays. When the first night was measured on a weekend (Friday), average total sleep time was significantly greater $(486 \pm 5 \mathrm{~min})$ compared with the second night (Saturday; $469 \pm 6 \mathrm{~min}), \mathrm{p}=0.01$.

Conclusions: We found no evidence to support a 'first night effect' for wrist actigraphy in our adolescent sample. The first night of actigraphy data should not be disregarded in future studies that deploy this technique to measure sleep over prolonged time periods.

\section{INTRODUCTION}

Polysomnography (PSG) is the gold standard method for the accurate determination of sleep and its quality. Laboratory PSG involves recordings via multiple electrodes and sensors that may disturb sleep and the
Strengths and limitations of this study

- This is the first time the possibility of a 'first night effect' for wrist actigraphy used to measure sleep has been investigated.

- A large sample of adolescents were instructed to wear the wrist actigraph for seven consecutive days, at baseline and then annually for 2 years.

- We compared multiple sleep parameters from the first night when the device was issued to all subsequent nights on both weekdays and weekends separately.

- The study findings are limited to healthy adolescents and may not be generalisable to other age groups, countries or specific patient populations.

recording may not reflect usual sleep. A key criticism of PSG is the 'first night effect'. Agnew et $a l^{1}$ observed a reduction in total sleep time, alterations to sleep architecture as well as poorer sleep quality on the first night of PSG assessment compared with the second. It is purported that multiple factors are responsible for these observations, including the equipment and the environment. ${ }^{2}$ Furthermore, more recent evidence suggests these effects may extend beyond the first night. ${ }^{2}$

The importance of sleep pertaining to health is increasingly recognised. Thus, establishing methods to accurately measure sleep, in the absence of a sleep laboratory, has been a recent focus. Advances in technology have made it possible to monitor sleep over prolonged periods in the absence of a laboratory. Sleep researchers are frequently using wrist actigraphy to monitor sleep-wake activity across multiple days/ nights. It has been recommended that five nights of actigraphy data may be required to enable an accurate representation of sleep, ${ }^{3}$ although this is inconsistent with others suggesting as many as seven consecutive nights in those with a potential sleep disorder, ${ }^{4}$ whereas some others have recommend a minimum of 72 hours actigraphy recording. ${ }^{5}$ 
It is possible, although currently unknown, if there is a 'first night effect' for wrist actigraphy when used to monitor sleep, as observed in PSG. If this effect does exist for wrist actigraphy, this has important implications for the design of future studies and data analysis.

Acebo $e t a l^{3}$ previously recommended at least five nights of valid actigraphy data is required for an accurate representation of sleep in paediatric populations. Up until now, it has remained unclear if the first night of actigraphy data can be used for sleep analysis or disregarded due to the possibility of the 'first night effect'. Clearly, if wrist actigraphy demonstrates a 'first night effect' then this would have important implications for future study design development, which includes actigraphy to monitor sleep as well as data analysis plans. The psychosocial aspects of recruitment to a study involving wrist actigraphy for sleep monitoring may subsequently influence sleep behaviour on the first night and beyond, before adaptation is achieved. Furthermore, wearing a sleep monitor may influence sleep on the first night until acclimatisation has been achieved. We therefore sought to examine potential differences between multiple sleep parameters between the first weekday night the wrist actigraph was worn compared with subsequent weekday nights in a large sample of adolescents.

\section{METHODS}

Nine secondary schools, from the Midlands region of the UK, were approached to participate in the Midlands Adolescents Schools Sleep Education Study (MASSES). Eight schools agreed to take part and were recruited to the study. Details of the MASSES have been previously described. ${ }^{6}{ }^{7}$ In brief, parents of students registered in year 7 or year 8 of participating schools were sent a letter regarding study participation during the first term of the 2011/2012 academic year. There was $80 \%$ parental response rate and 892 adolescents were eligible for study participation. Adolescent participants were included in the study if they had parental consent, provided personal assent, did not have a diagnosed sleep disorder, were not taking sleep medication, or had not travelled to a different time zone 4 weeks prior to data collection. All participants were aged between 11 and 13 years and registered in UK education at baseline. Study assessments were performed at baseline and annually thereafter for 2 years.

Of the total sample, students were randomly selected to wear the wrist actigraph (GT3X+, The ActiGraph, Florida, USA) at each annual assessment $(\mathrm{n}=332$ at baseline, $n=324$ at 1-year follow-up and $n=236$ at 2-year follow-up). Participants were mainly assessed in the second term of each academic year during term time. They were instructed to wear the actigraph on their nondominant wrist for seven consecutive days/nights at all times and the actigraph was issued on different weekdays (Monday-Friday) to different classes to accommodate school timetables and minimise disruption. Participants were instructed not to remove the device and were advised that the actigraph was waterproof. Data were downloaded using the manufacturer's software (ActiLife, V.5 or V.6). Corresponding sleep diaries, where completed, were used to indicate bedtimes and our group has previously shown good agreement between sleep diary reporting and wrist actigraphy. ${ }^{6}$ Sleep was automatically scored from $60 \mathrm{~s}$ epochs according to predefined algorithms. ${ }^{8}$ The Sadeh algorithm, adapted by the manufacturer, obtains data from the following: (1) arithmetic mean of activity counts for the window (AVG); (2) number of epochs that have counts $\geq 50$ and $<100$ (NATS); (3) SD for the first six epochs of the window (SD) and (4) natural (base e) logarithm of a current epoch (LG). The calculations are then used in the following equation:

$$
\begin{gathered}
(7.601-(0.065 \times \mathrm{AVG})-(1.08 \times \mathrm{NATS}) \\
-(0.056 \times \mathrm{SD})-(0.703 \times \mathrm{LG}))
\end{gathered}
$$

If the result of the algorithm was more than -4 , then epoch was scored as sleep.

Multiple sleep parameters were derived for each 24-hour period that the device collected data. These included: (1) total sleep time (minutes), indicating the total amount of sleep obtained; (2) wake after sleep onset (minutes), indicating the amount of time spent awake within each rest period; (3) sleep efficiency (\%) indicating the percentage of time spent asleep in a rest period; (4) sleep onset latency (minutes) indicating the length of time it took to initiate sleep; (5) number of awakenings indicating the number of awakenings an individual had in a given sleep period; (6) length of awakenings (minutes) indicating the average length of awakenings in a given sleep period and (7) time of sleep onset indicating the time that sleep was initiated. We compared all of the mean sleep variables obtained from the wrist actigraph, comparing those who were administered with the device on the first weekday (Monday), to all other subsequent weekdays (Tuesday, Wednesday and Thursday) that the device was worn. We further explored potential differences in sleep outcomes for when adolescents were given the device on a Tuesday compared with subsequent consecutive nights (Wednesday and Thursday). We then examined possible differences between those issued a wrist actigraph on a Wednesday and compared sleep outcomes on the next day (Thursday). Finally, we assessed significant differences in sleep variables between when the device was issued on a Friday and compared these data to Saturday night to determine potential 'first night effect' for weekend nights.

\section{Statistical analysis}

Analysis was performed using Stata (StataCorp. 2013) on all available data. Data are summarised as means (SE), unless otherwise stated. To compare sleep variables between two different nights, paired Student's t-tests 
were used for normally distributed variables (total sleep time and number of awakenings) and Wilcoxon Signed-rank test for skewed variables (wake after sleep onset, sleep efficiency, sleep onset latency and length of awakenings). Normality was assessed using Kolmogorov-Smirnov test. The $\mathrm{p}$ values $<0.05$ were considered to be significant.

\section{RESULTS}

At baseline, the mean age of the total sample was 12.0 \pm 0.7 years $(42.4 \%$ boys $)$. Table 1 highlights the mean (SE) of all sleep features obtained from the wrist actigraph across all weekdays. We show comparisons between sleep parameters when participants were issued a wrist actigraph on the first weekday (Monday) and compare these data to the subsequent weekday nights (Tuesday, Wednesday and Thursday). No significant differences were observed for any sleep measure between Monday (first night) with any other weekdays (Tuesday, Wednesday or Thursday), where $\mathrm{p} \geq 0.05$.

Table 2 shows comparisons between sleep variables for the first night of issue (Tuesday) and subsequent weekdays (Wednesday and Thursday). There were no significant differences in any sleep variable between the first night and the two subsequent nights that the actigraphy was worn.

The potential of a 'first night effect' was also assessed when participants were issued the wrist actigraphy on a Wednesday and sleep variables were compared with the next night (Thursday). Comparisons between all sleep features and the days are depicted in table 3, which highlights no significant differences for any sleep variable assessed, where $\mathrm{p} \geq 0.05$ for all.

We also report on differences in sleep outcomes when the first night was Friday (weekend) and compared these to the other weekend night (Saturday) (table 4). We observed a significant difference in average total sleep time on the first night (Friday) compared with the second night (Saturday), $486 \pm 5$ and $469 \pm 6$ min, respectively $(\mathrm{p}=0.01)$.

Table 5 depicts data concerning the number of usable nights of sleep data acquired from the wrist actigraph according to each annual assessment. The majority of participants had complete data from all seven nights that they were assessed.

\section{DISCUSSION}

We present novel findings from the first study to examine the possibility of a 'first night effect' for wrist actigraph used to monitor multiple sleep outcomes. We found no evidence of a 'first night effect' for any of the seven sleep features derived from actigraphy in a large sample of adolescents. The only significant difference observed was for total sleep time at the weekend when the first night (Friday) was, on average, $17 \mathrm{~min}$ longer compared with the second weekend night (Saturday).

Multiple studies have highlighted evidence to support the 'first night effect' for PSG in patient populations, ${ }^{9-12}$ healthy individuals ${ }^{2}{ }^{12-14}$ and in paediatrics. ${ }^{15}{ }^{16} \mathrm{~A}$ variety of possible explanations for this phenomenon have been linked to the environment as well as the equipment. PSG, unless the equipment is portable, requires the individual to sleep in an unfamiliar environment (sleep laboratory), where different conditions are likely to be encountered. These include external noises, different light exposure and room temperature, bed comfort and more. Given that a wrist actigraph permits the individual to sleep in their natural environment, these external alterations that may influence sleep outcomes are automatically controlled for and are therefore overcome. Thus, the possibility of a 'first night effect' for a wrist actigraph to monitor sleep is not likely to occur due to the environment, but rather the equipment and/ or psychosocial influences.

PSG is an intrusive technique, which has been shown to alter multiple sleep outcomes on the first night of

Table 1 Weekday differences in sleep features between Monday (first night) compared with all other weekdays

\begin{tabular}{|c|c|c|c|c|c|c|c|}
\hline Sleep measure & $\begin{array}{l}\text { Monday } \\
(n=95)\end{array}$ & $\begin{array}{l}\text { Tuesday } \\
(\mathrm{n}=94)\end{array}$ & $\begin{array}{l}p \\
\text { Value }\end{array}$ & $\begin{array}{l}\text { Wednesday } \\
(n=94)\end{array}$ & $\begin{array}{l}\mathbf{p} \\
\text { Value }\end{array}$ & $\begin{array}{l}\text { Thursday } \\
(n=93)\end{array}$ & $\begin{array}{l}\mathbf{p} \\
\text { Value }\end{array}$ \\
\hline TST (minutes) ${ }^{\star}$ & $440(5)$ & $437(7)$ & 0.65 & $443(10)$ & 0.77 & $449(10)$ & 0.32 \\
\hline WASO (minutes) $†$ & $69(45)$ & $71(55)$ & 0.88 & $67(59)$ & 0.72 & $67(44)$ & 0.98 \\
\hline Sleep efficiency $(\%) \dagger$ & 86.1 (9.3) & $86.3(10.5)$ & 0.84 & $87.1(11.6)$ & 0.86 & $86.5(9.6)$ & 0.70 \\
\hline SOL (minutes) $\dagger$ & $2(4)$ & $1(4)$ & 0.63 & $2(4)$ & 0.36 & $2(4)$ & 0.82 \\
\hline Number of awakenings* & $23(1)$ & $22(1)$ & 0.29 & $21(1)$ & 0.07 & $21(1)$ & 0.05 \\
\hline $\begin{array}{l}\text { Length of awakenings } \\
\text { (minutes) } \dagger\end{array}$ & $3(1)$ & $3(1)$ & 0.12 & $3(2)$ & 0.34 & $3(1)$ & 0.11 \\
\hline \multirow[t]{3}{*}{ Sleep onset time } & & S: $9.1 \%$ & & S: $10.2 \%$ & & S: $9.1 \%$ & \\
\hline & & L: $46.6 \%$ & & L: $54.6 \%$ & & L: $52.3 \%$ & \\
\hline & & E: $44.3 \%$ & & E: $35.2 \%$ & & E: $38.6 \%$ & \\
\hline
\end{tabular}

$\mathrm{E}=$ more than $30 \mathrm{~min}$ earlier than Monday; $\mathrm{L}=$ more than $30 \mathrm{~min}$ later than Monday; $\mathrm{S}=$ within $30 \mathrm{~min}$ of Monday.

${ }^{*}$ Data are presented as mean (SE), paired Student's t-test.

†Data are presented as median (IQR), Wilcoxon Signed-rank test.

SOL, sleep onset latency; TST, total sleep time; WASO, wake after sleep onset. 
Table 2 Weekday differences in sleep features between Tuesday (first night) and all other weekdays

\begin{tabular}{|c|c|c|c|c|c|}
\hline Sleep measure & Tuesday $(n=161)$ & Wednesday $(n=159)$ & p Value & Thursday $(n=159)$ & p Value \\
\hline TST (minutes)* & $452(5)$ & $462(5)$ & 0.06 & $446(4)$ & 0.28 \\
\hline WASO (minutes) $\dagger$ & $64(39)$ & $60(43)$ & 0.20 & $58(44)$ & 0.26 \\
\hline Sleep efficiency (\%)† & $87.4(7.1)$ & $88.1(6.9)$ & 0.07 & $87.7(8.6)$ & 0.32 \\
\hline $\mathrm{SOL}$ (minutes) $\dagger$ & $2(4)$ & $2(4)$ & 0.38 & $2(4)$ & 0.31 \\
\hline Number of awakenings ${ }^{*}$ & $22(1)$ & $21(1)$ & 0.33 & $22(1)$ & 0.83 \\
\hline Length of awakenings (minutes) $\dagger$ & $3.0(1.1)$ & $2.8(1.3)$ & 0.16 & $2.8(1.3)$ & 0.06 \\
\hline \multirow[t]{3}{*}{ Sleep onset time } & & S: $15.8 \%$ & & S: $12.2 \%$ & \\
\hline & & L: $47.5 \%$ & & L: $54.0 \%$ & \\
\hline & & E: $36.7 \%$ & & E: $33.8 \%$ & \\
\hline
\end{tabular}

$E=$ more than 30 min earlier than Tuesday; $L=$ more than 30 min later than Tuesday; $S=$ within 30 min of Tuesday.

*Data are presented as mean (SE), paired Student's t-test.

†Data are presented as median (IQR), Wilcoxon Signed-rank test.

SOL, sleep onset latency; TST, total sleep time; WASO, wake after sleep onset.

Table 3 Weekday differences in sleep features between Wednesday (first night) compared with Thursday

\begin{tabular}{lccc}
\hline Sleep measure & Wednesday $(\mathbf{n}=\mathbf{1 8 5})$ & Thursday $(\mathbf{n = 1 8 8})$ & p Value \\
\hline TST (minutes)* & $442(4)$ & $437(4)$ & 0.20 \\
WASO (minutes) $\dagger$ & $68(43)$ & $65(46)$ & 0.40 \\
Sleep efficiency (\%)† & $86.1(9.3)$ & $87.1(7.5)$ & 0.54 \\
SOL (minutes) $\dagger$ & $2(4)$ & $2(4)$ & 0.38 \\
Number of awakenings* & $24(1)$ & $22(1)$ & 0.05 \\
Length of awakenings (minutes) $\dagger$ & $3(2)$ & $3(1)$ & 0.96 \\
Sleep onset time & & S: $9.4 \%$ & \\
& & L: $52.4 \%$ & \\
\hline
\end{tabular}

$\mathrm{E}=$ more than 30 min earlier than Wednesday; $\mathrm{L}=$ more than 30 min later than Wednesday; $\mathrm{S}=$ within 30 min of Wednesday.

*Data are presented as mean (SE), paired Student's t-test.

†Data are presented as median (IQR), Wilcoxon Signed-rank test.

SOL, sleep onset latency; TST, total sleep time; WASO, wake after sleep onset.

Table 4 Weekend differences in sleep features between Friday (first night) and Saturday

\begin{tabular}{|c|c|c|c|}
\hline Sleep measure & Friday $(n=169)$ & Saturday $(n=168)$ & p Value \\
\hline TST (minutes) ${ }^{\star}$ & $486(5)$ & $469(6)$ & 0.01 \\
\hline WASO (minutes)† & $73(52)$ & $72(41)$ & 0.50 \\
\hline Sleep efficiency $(\%) \dagger$ & $85.9(7.7)$ & $86.1(8.1)$ & 0.84 \\
\hline SOL (minutes) $\dagger$ & $3(4)$ & $2(4)$ & 0.19 \\
\hline Number of awakenings* & $25(1)$ & $25(1)$ & 0.86 \\
\hline Length of awakenings (minutes) $\dagger$ & $3(1)$ & $3(1)$ & 0.39 \\
\hline \multirow[t]{3}{*}{ Sleep onset time } & & S: $8.5 \%$ & \\
\hline & & L: $61.5 \%$ & \\
\hline & & E: $30.0 \%$ & \\
\hline $\begin{array}{l}\mathrm{E}=\text { more than } 30 \text { min earlier than Frida) } \\
{ }^{*} \text { Data are presented as mean (SE), pa } \\
\dagger \text { Data are presented as median (IQR), }\end{array}$ & $\begin{array}{l}\text { min later than Frid } \\
\text { st. } \\
\text {-rank test. }\end{array}$ & in of Friday. & \\
\hline
\end{tabular}

sleep assessment as compared with subsequent nights. ${ }^{1} 2{ }^{2}$-16 Reasons for this include restriction of movement and awkwardness about the equipment, which may cause unrest and hyperarousal as a result of the PSG equipment. Despite wrist actigraphy being much less invasive than PSG, the presence of equipment to monitor sleep may alter sleep outcomes through physical and psychological effects. For example, the knowledge of being entered into a research study where sleep is monitored using a wrist actigraph may subsequently influence sleep behaviours via psychological mechanisms. Wearing the device may result in individuals purposely intending to alter sleep behaviours (until acclimatisation is achieved), to either distort the 
Table 5 Number of nights of valid actigraphy data according to each annual assessment

\begin{tabular}{|c|c|c|c|c|c|c|c|}
\hline \multirow[b]{2}{*}{ Time point } & \multicolumn{7}{|c|}{ Number of nights of valid data } \\
\hline & 1 night & 2 nights & 3 nights & 4 nights & 5 nights & 6 nights & 7 nights \\
\hline Baseline & 6 & 4 & 8 & 11 & 7 & 14 & 508 \\
\hline Year 1 & 13 & 8 & 9 & 11 & 19 & 37 & 227 \\
\hline Year 2 & 11 & 12 & 9 & 27 & 15 & 30 & 132 \\
\hline
\end{tabular}

data or have more socially desirable sleep outcomes, knowing that sleep is being investigated. If these intentional sleep behaviour alterations occur then this will result in misrepresentation of the sleep outcomes assessed. The physical presence of a wrist actigraph may paradoxically contribute to sleep alterations due to discomfort, particularly if the individual does not habitually sleep while wearing a wrist device. Furthermore, depending on the type of actigraphy and the initialisation process, some can emit light to indicate that the device is collecting data, which is likely to cause sleep disruption. The aforementioned arguments support the potential 'first night effect' for wrist actigraphy for sleep assessment. Contrary to PSG findings, our wrist actigraphy data showed no evidence to support a 'first night effect'. Factors relating to the sleep environment and equipment, which may be responsible for differences between the first night and subsequent nights of PSG assessment, were not shown to influence subsequent sleep behaviour in adolescents wearing a wrist actigraph.

The findings from our study indicated a significant difference for total sleep time at the weekend when the first night was a Friday, compared with the Saturday. The difference, although statistically significant, was an average of just $17 \mathrm{~min}$ more on the first night. This can be explained by the possibility of accumulated weekday sleep debt (discrepancy between an individuals sleep need and the amount of sleep actually achieved), which is repaid at the first opportunity (eg, the first weekend night). Seventeen minutes of additional sleep is not, however, likely to compensate for the amount of sleep debt that a typical adolescent accrues across the school week. For example, Kim et $a l^{17}$ found that adolescents sleep, on average, 2 hours and $42 \mathrm{~min}$ less on weekdays compared with weekend nights. On closer inspection, there was no evidence of weekend catch-up sleep in our adolescent cohort where the average sleep quantity and quality did not differ during the week compared with weekends.

While our study is the first to investigate the possibility of a 'first night effect' for wrist actigraphy on sleep outcomes, we acknowledge several limitations. First, we combined data from participants across three study time points. Repeated exposure to wrist actigraphy may have resulted in participants becoming more acclimatised to wearing the device during the second and third assessment. Furthermore, we repeated our analysis in the baseline data only and the results remained consistent (data not shown), suggesting acclimatisation may not occur with annual assessment. Second, our findings may not be generalisable to other populations. For example, different age groups and patient populations, particularly those with sleep disorders, may exhibit different patterns and could be the focus of future studies. Third, while actigraphy benefits from participants being in their habitual environment, there was no way of determining if participants remained in their own sleep environment or at another location (ie, friend, relative, hotel). We also have no way of determining if participants were subjected to external sleep disruptors such as pets, which could have influenced the output. Finally, while we recognise that multiple types of wrist actigraphs have been validated against PSG for multiple sleep outcomes in many groups, ${ }^{18-32}$ not all sleep parameters included in our study have been validated. It remains a possibility that some differences may be present between the first night and subsequent nights according to the actigraph type/manufacture, given that multiple devices are now available.

In conclusion, we found no evidence to support a 'first night effect' for wrist actigraphy to monitor sleep in a large cohort of adolescents. The first night of actigraphy data collected to measure sleep should be included and not disregarded at this stage. However, further efforts are required to determine if these findings are consistent in different age groups and/or patient populations and with the application of different types of wrist actigraphs that are currently available and used for sleep research.

Acknowledgements The authors thank all students for their participation and parents who gave consent to their child to take part in the study. They specially thank the teachers who supported the study-Claire Horne, Jess Sheridan, Stuart Ingleston-Orme, Tim Owens, Richard Merriman, Ami Hands, Inderpal Kalsi, Jane Taylor and Denyse Price.

Contributors TA and ST made substantial contributions to the conception and design of the study. TA acquired the study data. OMO performed data analysis. All authors contributed to interpretation of the study results. TA wrote the first draft and the other two authors critically revised it for important intellectual content. All authors have approved the final version of the manuscript and agree to be accountable for all aspects of the work performed.

Funding This work was supported by the children's charity, Action Medical Research, grant number SP4609.

Competing interests None declared.

Patient consent Obtained.

Ethics approval University of Birmingham Research Ethics Committee (ERN_08-437). 
Provenance and peer review Not commissioned; externally peer reviewed.

Data sharing statement No additional data are available.

Open Access This is an Open Access article distributed in accordance with the Creative Commons Attribution Non Commercial (CC BY-NC 4.0) license, which permits others to distribute, remix, adapt, build upon this work noncommercially, and license their derivative works on different terms, provided the original work is properly cited and the use is non-commercial. See: http:// creativecommons.org/licenses/by-nc/4.0/

\section{REFERENCES}

1. Agnew HW Jr, Webb WB, Williams RL. The first night effect: an EEG study of sleep. Psychophysiology 1966;2:263-6.

2. Le Bon O, Staner L, Hoffmann G, et al. The first-night effect may last more than one night. J Psychiatr Res 2001;35:165-72.

3. Acebo C, Sadeh A, Seifer R, et al. Estimating sleep patterns with activity monitoring in children and adolescents: how many nights are necessary for reliable measures? Sleep 1999;22:95-103.

4. Martin JL, Hakim AD. Wrist actigraphy. Chest 2011;139:1514-27.

5. Littner M, Kushida CA, Anderson WM, et al. Practice parameters for the role of actigraphy in the study of sleep and circadian rhythms: an update for 2002. Sleep 2003;26:337-41.

6. Arora T, Broglia E, Pushpakumar D, et al. An investigation into the strength of the association and agreement levels between subjective and objective sleep duration in adolescents. PLOS ONE 2013;8: e72406.

7. Arora $\mathrm{T}$, Taheri $\mathrm{S}$. Associations among late chronotype, body mass index and dietary behaviors in young adolescents. Int $J$ Obes (Lond) 2015;39:39-44.

8. Sadeh A, Sharkey KM, Carskadon MA. Activity-based sleep-wake identification: an empirical test of methodological issues. Sleep 1994;17:201-7.

9. Le Bon O, Minner P, Van Moorsel C, et al. First-night effect in the chronic fatigue syndrome. Psychiatry Res 2003;120:191-9.

10. Marzec ML, Selwa LM, Malow BA. Analysis of the first night effect and sleep parameters in medically refractory epilepsy patients. Sleep Med 2005;6:277-80.

11. Rotenberg VS, Hadjez J, Kimhi R, et al. First night effect in depression: new data and a new approach. Biol Psychiatry 1997:42:267-74.

12. Toussaint $M$, Luthringer $R$, Schaltenbrand $N$, et al. First-night effect in normal subjects and psychiatric inpatients. Sleep 1995;18:463-9.

13. Lorenzo JL, Barbanoj MJ. Variability of sleep parameters across multiple laboratory sessions in healthy young subjects: the "very first night effect". Psychophysiology 2002;39:409-13.

14. Moser D, Kloesch G, Fischmeister FP, et al. Cyclic alternating pattern and sleep quality in healthy subjects-is there a first-night effect on different approaches of sleep quality? Biol Psychol 2010;83:20-6.

15. Scholle S, Scholle HC, Kemper A, et al. First night effect in children and adolescents undergoing polysomnography for sleep-disordered breathing. Clin Neurophysiol 2003;114:2138-45.
16. Verhulst SL, Schrauwen N, De Backer WA, et al. First night effect for polysomnographic data in children and adolescents with suspected sleep disordered breathing. Arch Dis Child 2006;91:233-7.

17. Kim SJ, Lee YJ, Cho SJ, et al. Relationship between weekend catch-up sleep and poor performance on attention tasks in Korean adolescents. Arch Pediatr Adolesc Med 2011;165:806-12.

18. Baandrup L, Jennum PJ. A validation of wrist actigraphy against polysomnography in patients with schizophrenia or bipolar disorder. Neuropsychiatr Dis Treat 2015;11:2271-7.

19. Belanger ME, Bernier A, Paquet J, et al. Validating actigraphy as a measure of sleep for preschool children. J Clin Sleep Med 2013;9:701-6.

20. de Souza L, Benedito-Silva AA, Pires ML, et al. Further validation of actigraphy for sleep studies. Sleep 2003;26:81-5.

21. de Zambotti M, Baker FC, Colrain IM. Validation of sleep-tracking technology compared with polysomnography in adolescents. Sleep 2015;38:1461-8.

22. de Zambotti M, Baker FC, Willoughby AR, et al. Measures of sleep and cardiac functioning during sleep using a multi-sensory commercially-available wristband in adolescents. Physiol Behav 2016;158:143-9.

23. Dick R, Penzel T, Fietze I, et al. AASM standards of practice compliant validation of actigraphic sleep analysis from SOMNOwatch versus polysomnographic sleep diagnostics shows high conformity also among subjects with sleep disordered breathing. Physiol Meas 2010;31:1623-33.

24. Hyde M, O'Driscoll DM, Binette S, et al. Validation of actigraphy for determining sleep and wake in children with sleep disordered breathing. J Sleep Res 2007;16:213-16.

25. Kanady JC, Drummond SP, Mednick SC. Actigraphic assessment of a polysomnographic-recorded nap: a validation study. J Sleep Res 2011;20(1 Pt 2):214-22.

26. Kosmadopoulos A, Sargent C, Darwent D, et al. Alternatives to polysomnography (PSG): a validation of wrist actigraphy and a partial-PSG system. Behav Res Methods 2014;46:1032-41.

27. Lichstein KL, Stone KC, Donaldson J, et al. Actigraphy validation with insomnia. Sleep 2006;29:232-9.

28. Marino M, Li Y, Rueschman MN, et al. Measuring sleep: accuracy, sensitivity, and specificity of wrist actigraphy compared to polysomnography. Sleep 2013;36:1747-55.

29. Meltzer LJ, Walsh CM, Traylor J, et al. Direct comparison of two new actigraphs and polysomnography in children and adolescents. Sleep 2012;35:159-66.

30. Sadaka Y, Sadeh A, Bradbury L, et al. Validation of actigraphy with continuous video-electroencephalography in children with epilepsy. Sleep Med 2014;15:1075-81.

31. Shin M, Swan P, Chow CM. The validity of Actiwatch2 and SenseWear armband compared against polysomnography at different ambient temperature conditions. Sleep Sci 2015;8: 9-15.

32. So K, Buckley P, Adamson TM, et al. Actigraphy correctly predicts sleep behavior in infants who are younger than six months, when compared with polysomnography. Pediatr Res $2005 ; 58: 761-5$ 